\title{
Cytokine Storm
}

\author{
Satyabrata Ganguly ${ }^{1}$, Tanuka Mandal ${ }^{2}$
}

\begin{abstract}
Cytokine storm is said to occur when increased levels of cytokines are disproportionately produced, with systemic effect and collateral damage to vital organs. It can be caused by both intrinsic (autoimmune) or extrinsic (COVID-19) diseases. In the absence of pathogens, cytokine storm can occur due to immune hyperactivation as a result of inappropriate triggering. Interleukin-6, tumor necrosis factor $\alpha$, and interleukin-18 are cytokines of clinical importance in cytokine storm. Interleukin-18, a biomarker of severity, correlates with hyperferritinemia and disease flare. The underlying condition should be detected and treatment should be individualized.

Keywords: Biomarker, COVID-19, Cytokines.

Bengal Physician Journal (2020): 10.5005/jp-journals-10070-7037
\end{abstract}

\section{INTRODUCTION}

The term cytokine storm was first used in 1993 to describe graft versus host disease after allogeneic hematopoietic stem cell transplantation. ${ }^{1}$ In a number of conditions like various therapies [chimeric antigen receptor CAR T cell] therapy, pathogens, cancers, autoimmune conditions, and monogenic disorders cause hyperimmune response and release of excess cytokines from macrophages, which is a devastating condition.

Cytokine storm can be caused by both intrinsic (autoimmune) or extrinsic (COVID-19) diseases. So it is difficult to describe it by a single definition. ${ }^{2}$ The cytokines released from macrophages and interleukin-1, interleukin-6, tumor necrosis factor $\alpha$ (TNF- $\alpha$ ), and interleukin-18. T cells and NK cells secrete interferon- $\gamma$ before antigen-specific interferon- $\gamma$ is produced by T cells. ${ }^{3}$ Fever, chills, headache, dizziness, and fatigue can be caused by interferon- $\gamma$. Emapalumab, a monoclonal antibody that binds interferon- $\gamma$ is now used to treat cytokine storm with primary hemophagocytic lymphohistiocytosis (HLH).

It is very important to distinguish cytokine storm from iatrogenic disorder (CAR T cell) or from sepsis because immunosuppression which is a treatment for the former will be dangerous for sepsis. In CAR T-cell therapy, the level of interferon- $\gamma$ is very much elevated but in sepsis-induced cytokine storm, interleukin- $1 \beta$ and procalcitonin are increased.

\section{Clinical Features}

- Fever-almost in all cases may be of high grade. ${ }^{4}$

- Fatigue, myalgia, anorexia, rash, headache, diarrhea, arthralgia with neuropsychiatric findings.

The causes of these symptoms are

- Cytokine-induced tissue damage

- Acute phase physiological changes

- Immune cell-medicated response

The patient of cytokine storm frequently suffers from hemorrhage due to hyperinflammation, coagulopathy, and low platelet counts.

The severe cases of cytokine storm lead to Takotsubo like cardiomyopathy and acute liver and kidney injuries. ${ }^{4}$ Capillary leak
${ }^{1}$ Department of Medicine, KPC Medical College and Hospital, Kolkata, West Bengal, India

${ }^{2}$ Department of Medicine, RG Kar Medical College, Kolkata, West Bengal, India

Corresponding Author: Tanuka Mandal, Department of Medicine, RG Kar Medical College, Kolkata, West Bengal, India, Phone: +91 8240769830, e-mail: tanukamandal@ymail.com

How to cite this article: Ganguly S, Mandal T. Cytokine Storm. Bengal Physician Journal 2020;7(3):70-71.

Source of support: Nil

Conflict of interest: None

syndrome can also occur due to renal dysfunction, endothelial cell death, and acute phase hypoalbuminemia.

\section{InVESTIGATIONS}

- Increase in CRP (c-reactive protein)-a universal finding

- Leukocytosis, high triglyceride levels, thrombocytopenia, high serum ferritin is also seen.

- Elevation of serum inflammatory cytokines, that is, interferon- $\gamma$, interleukin- 6 and interleukin-10 with soluble interleukin-2 receptor $\alpha$ (a marker of T-cell activation) is usually present.

\section{Goals of Evaluation}

- Identification of underlying disorder causing cytokine storm

- Establishing the severity of the condition

- Excluding the conditions which may mimic cytokines storm like anaphylaxis which should be ruled out

In our body, the immune system recognizes foreign invaders and by proportionate response neutralizes it. Cytokines produced by macrophages are the main player in our defence ${ }^{5}$ but as they have short lives, they cannot produce any effect outside the site of inflammation. When increased levels of cytokines are disproportionately produced, the systemic effect may occur and cause collateral damage to vital organs. In the absence of pathogens, cytokine storm can occur due to immune hyperactivation as a result of inappropriate triggering. 
Cytokine storm involves an immune response that causes collateral damage which may be greater than the benefit of the immune response.

Interleukin-6, TNF- $\alpha$ and interleukin-18 are cytokines of clinical importance in cytokine storm. Interleukin-18, a biomarker of severity that correlates with hyperferritinemia me near and disease flare. Interleukin-18 Binding Protein prevents the binding of interleukin-18 to its receptor thus prevents its proinflammatory action.

In cytokine storm induced by microbial infection, the collateral damage can lead to fever, cell death, coagulopathy, and multiorgan dysfunction. The damage caused by immune reactions to clear the organisms is more harmful than microbial damage.

Disseminated viral infections can also induce profound cytokines storm. Targeted treatment is more challenging in viral infections as there are few antiviral drugs.

In patients with primary $\mathrm{HLH}$, various autosomal recessive monogenic abnormalities in granule-mediated cytotoxicity lead to cytotoxic storm. ${ }^{6}$

\section{Drugs Effective in Cytotoxic Storm}

- Glucocorticoids

- Cyclosporin

- Interleukin-1 antibody

- $\mathrm{JAK}_{1}$ and $\mathrm{JAK}_{2}$ inhibitors

- Interleukin-6 inhibitors

- Cyclophosphamide and etoposide which are broadly cytotoxic but effective in eliminating activated CD $8+T$ cells

- Sirolimus in moderate cytokine storm

\section{COVID-19 And Cytokine Storm}

COVID-19 is an important cause of cytokine storm, here interleukin-1 $\beta$, interleukin- 6 , TNF- $\alpha$, interferon- $\gamma$, macrophage inflammatory protein, vascular endothelial growth factor are increased. Shorter survival is associated with higher IL-6 levels.

Increased levels of CD4+ CD8+ " $T$ " cells are also seen. The most interesting fact is inadequate viral clearance and hyperimmune response to viral load both may be culprit in different cases. Coexisting conditions such as hypertension, diabetes, and obesity are associated with severity-probably due to the persistence of chronic inflammatory state in this patient.

Certain issues need clarification and discussion in COVID-19induced cytokine storm.

- Cytokine storm triggered by infection of COVID-19 helps to reduce viral load so anticytokine therapy, i.e., Tocilizumab against IL-6, is not helpful in COVID-19 infection. So here cytokine (IL-6) is helpful for the host as it reduces viral load.

- In HHV-8 associated Castleman disease selective elimination of the primary viral load is beneficial but not possible with COVID-19.

- Lymphopenia is a bad prognostic sign in COVID-19 infection but is not often seen in other viral infections. The cause of lymphopenia is not clear but may be due to the destruction of lymphocytes or due to tissue infiltration.

- Clotting issues can occur in all cytokines storm but thromboembolic events are more common in COVID-19.

Though the total picture is not fully clear, many immunomodulating drugs, that is, Canakinumab, anti-interleukin-1 $\beta$ monoclonal antibody, and Anakinra, are both being studied for COVID-19-introduced acute respiratory distress syndrome.

\section{TREATMENT}

- Supportive care to maintain critical organ function, control the underlying disease, and elimination of triggers.

- The paradox which needs explanation is that the neutralization of cytokines whose level is high by anti-interleukin- 6 , TNF- $\alpha$, anti-interferon- $\gamma$ or anti-interleukin-1 $\beta$ will not always be effective on the contrast, blocking a key cytokine whose level is normal may sometimes be effective.

- $\mathrm{JAK}_{1}$ inhibitors are more important investigating drugs where multiple cytokines receptor pairs can be targeted simultaneously.

- Plasma exchange and plasma filtration columns for adsorption of cytokines or both under evolution for cytokine disorders.

\section{CONCLUSION}

Inflammation is the reaction of the body against injury, injury may be due to an external agent (infection) or due to the body's own antigen(autoimmune disease) and cytokines are the mediators of inflammation. If the damage caused by these cytokines causes the secondary multiple organ dysfunction to such a dangerous level so that the host survival is at risk, then it is pathological and may be called cytokine storm, when levels of cytokines are high. Proper understanding of pathophysiology of such conditions and appropriate treatment can only save the host.

\section{References}

1. Clark IA. The advent of the cytokine storm. Immunol Cell Biol 2007;85(4):271-273. DOI: 10.1038/sj.icb.7100062. PMID: 17551531.

2. Costela-Ruiz VJ, Illescas-Montes R, Puerta-Puerta JM, Ruiz C, MelguizoRodríguez L. SARS-CoV-2 infection: the role of cytokines in COVID-19 disease. Cytokine Growth Factor Rev 2020;54:62-75. DOI: 10.1016/j. cytogfr.2020.06.001. PMID: 32513566; PMCID: PMC7265853.

3. Mah AY, Cooper MA. Crit Rev Immunol 2016;36(2):131-147. DOI: 10.1615/CritRevImmunol.2016017387.

4. Fajgenbaum DC, June CH. Cytokine storm. N Engl J Med 2020;383(23):2255-2273. DOI: 10.1056/NEJMra2026131. PMID: 33264547; PMCID: PMC7727315.

5. Arango Duque G, Descoteaux A. Macrophage cytokines: involvement in immunity and infectious diseases. Front Immunol 2014;5:491. DOI: 10.3389/fimmu.2014.00491. PMID: 25339958; PMCID: PMC4188125.

6. Schulert GS, Grom AA. Macrophage activation syndrome and cytokine-directed therapies. Best Pract Res Clin Rheumatol 2014;28(2):277-292. DOI: 10.1016/j.berh.2014.03.002. PMID: 24974063; PMCID: PMC4074772. 